\title{
Protokollempfehlungen der AG Bildgebende Verfahren des Bewegungsapparates (AG BVB) der Deutschen Röntgengesellschaft (DRG) zu Messsequenzen für die Gelenk-MRT
}

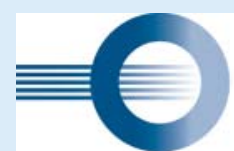

AC Bildgebende Verfahren des Bewegungsapparates

in der Deutschen Röntgengesellschaft

\section{Allgemeine Empfehlungen}

- Jedes Gelenk sollte immer in drei Ebenen untersucht werden.

- Spezialebenen sind in manchen Bereichen sinnvoll (siehe spezielle Empfehlungen):

- Bei der Messung sollte in den meisten Fällen zunächst die axiale Sequenz senkrecht in zwei Ebenen auf den angrenzenden Röhrenknochen geplant werden. Auf der axialen Sequenz können dann die Koronare und sagittale Messung exakter als auf den Topos geplant werden.

\section{Art der Messsequenzen}

- In der Regel sollten Spin-Echo-Sequenzen mit Turbobeschleunigung gemessen werden. Neben der kürzeren Messzeit wird hierdurch die Robustheit gegenüber Auslöschartefakte bei Metallimplantaten oder Metallabrieb erhöht.

- Je schneller eine Sequenz bei guter Qualität gemessen werden kann, desto geringer ist die Gefahr von Bewegungsartefakten. Falls hardware- und softwareseitig möglich, sollte daher mit paralleler Technik (GRAPPA, SENSE) gemessen werden.

- Bei unruhigen Patienten sollte frühzeitig auf Sequenzen mit nicht linearer Abtastung des k-Raums (Propeller, BLADE) umgestiegen werden.
- Bei PD-gewichteten Sequenzen sollte mit einem TE > 40 ms zur Vermeidung des Magic-Angle-Phänomens gemessen werden.

- Zur Beurteilung des Knochenmarks sollte mindestens eine Ebene T1-gewichtet gemessen werden.

- Die Sensitivität für frische Verletzungen an Bändern, Sehnen und Muskeln, für Knochenmarkveränderungen und die Knorpelbeurteilung wird durch Fettsättigungstechniken verbessert. Daher sollten mindestens 2 Ebenen mit Fettsättigung gemessen werden.

- Signalarme Strukturen, wie Fibrosen und Bänder sind auf dunklem Hintergrund schwer oder nicht erkennbar. Daher ist für die Beurteilung dieser Strukturen eine Sequenz ohne Fettsättigung von Vorteil.

- Gradientenechosequenzen, wie z. B. die True FISP Sequenz mit Water Excitation, können für die Knorpelbeurteilung hilfreich sein.

- 3 D Sequenzen können in manchen Fällen additiv gemessen werden. Sie können jedoch derzeit 2D-Sequenzen nicht ersetzen:

\section{Auflösung}

Auflösung: Oft wird bei Empfehlungen das FOV angegeben. Dieser Wert ist aber nur in Verbindung mit der Matrix sinnvoll. Um Details beurteilen zu können, muss die gemessene In-plane-Auflösung auf die Größe des
Gelenks bzw. der Gelenkstrukturen angepasst werden. Als Richtschnur sollte sie bei großen Gelenken (Hüfte einseitig, Schulter) mindestens $1,0 \mathrm{~mm} \times 1,0 \mathrm{~mm}$, bei mittelgroßen Gelenken (Knie, Sprunggelenk, Ellbogen) mindestens $0,6 \mathrm{~mm} \times 0,6 \mathrm{~mm}$ und bei kleinen Gelenken mindestens (Handgelenk, Finger) $0,4 \mathrm{~mm} \times 0,4 \mathrm{~mm}$ betragen . Die Schichtdicke sollte bei großen und mittelgroßen Gelenken $\leq 3,5 \mathrm{~mm}$, bei kleinen Gelenken (Hand/Handgelenk/Fuß) $\leq 2,5 \mathrm{~mm}$ sein.

Wegen der größeren geforderten Auflösung bei kleinen Gelenken, sollten diese bevorzug an Hochfeld-Scannern (3 T) und/oder mit dezidierten Spulen gemessen werden.

\section{Kontrastmittel}

- i. v. Kontrastmittel: Bei einer akuten Verletzung ist die Kontrastmittelgabe in der Regel entbehrlich. Bei chronischen Schmerzen und bei der Frage nach entzündlichen Veränderungen wird die Kontrastmittelgabe empfohlen.

- intraartikuläres Kontrastmittel: manche Strukturen sind in einer konventionellen MRT unauffällig und werden erst durch das Eindringen von Flüssigkeit/Kontrastmittel in Band- oder Muskellücken (z. B. SL-Band, Supraspinatusläsion), Spalten und Rissen (z. B. SLAP Läsion, instabile OD oder Labrumläsionen) oder durch umspülen (freie Gelenkkörper) sichtbar. In diesen Fällen, insbesondere wenn schon eine unauffällige konventionelle MRT vorliegt, sollte eine MRT mit intraartikulären Kontrastmittel (ArthroMRT) angestrebt werden. 


\section{Spezielle Empfehlungen}

\section{Obere Extremität}

\section{Schulter}

Lagerung Rückenlage, Kopf zuerst, Arm parallel zum Tisch, Handfläche nach oben (Supinationstellung), evtl. mit Sandsack stabilisieren.

\begin{tabular}{|c|c|c|}
\hline Sequenz & Planung & Kommentar \\
\hline ax PD fs & $\begin{array}{l}\text { das Akromion muss auf der obersten Schicht enthalten sein, in } \\
\text { beiden Ebenen senkrecht zum Humerusschaft planen }\end{array}$ & auf Os acromiale achten \\
\hline cor PD/T2 fs & parallel zur Sehne des M. supraspinatus und zum Humerusschaft & $\begin{array}{l}\text { TE > } 60 \text { ms wählen um das Magic-angle-Phänomen an der } \\
\text { Supraspinatussehne zu vermeiden }\end{array}$ \\
\hline sag T1 & $\begin{array}{l}\text { senkrecht zu cor, parallel zum Humerusschaft, bis zum Scapula-Y } \\
\text { nach medial messen }\end{array}$ & $\begin{array}{l}\text { nicht fettsättigen, um fibröses Gewebe im Rotatorenintervall } \\
\text { nicht zu übersehen }\end{array}$ \\
\hline sag PD fs & & $\begin{array}{l}\text { ist verzichtbar, hilft aber zur Beurteilung von Ödem in den } \\
\text { Muskelbäuchen der Rotatorenmanschette, z. B. bei Denervierung }\end{array}$ \\
\hline ggf. axial GRE & & kann die Beurteilung des Labrums verbessern \\
\hline ax und cor $\mathrm{T} 1 \mathrm{fs} \mathrm{KM}$ & & $\begin{array}{l}\text { erhöht die Sensitivität für Muskel- und Sehnenverletzungen und } \\
\text { Entzündungen, z. B. Capsulitis }\end{array}$ \\
\hline
\end{tabular}

\section{Arthro-Schulter}

Indikation Die klassische Indikation ist der unklare anteriore Schulterschmerz und die Instabilität bei jungen Patienten. Sie dient der besseren Beurteilung des Labrums und des Bizepsankers und hat wohl auch eine höhere Sensitivität für Verletzungen der Rotatorenmanschette, insbesondere von humerusseitigen Verletzungen des M. supraspinatus. Bei frischen Luxationen kann bei Vorliegen eines gesicherten Gelenkergusses auf eine Arthrografie verzichtet werden. Im Messprotokoll müssen dann aber die T1-gewichteten Sequenzen durch PD oder T2-gewichtete Sequenzen ersetzt werden.

Lagerung Rückenlage, Kopf zuerst, Arm parallel zum Tisch, Handfläche nach oben Supinationstellung), evtl. mit Sandsack stabilisieren.
Lagerung ABER Position Handfläche unter den Kopf, Oberarm und Rumpf bilden ca. einen $90^{\circ}$ Winkel.

\begin{tabular}{|c|c|c|}
\hline Sequenz & Planung & Kommentar \\
\hline $\mathrm{ax} \mathrm{T} 1 \mathrm{fs}$ & $\begin{array}{l}\text { das Akromion muss auf den obersten Schichten enthalten } \\
\text { sein, senkrecht zum Humerusschaft }\end{array}$ & \\
\hline cor PD/T2 fs & $\begin{array}{l}\text { parallel zur Sehne des M. supraspinatus und zum Humerus- } \\
\text { schaft }\end{array}$ & $\begin{array}{l}\text { TE > } 60 \text { ms wählen um sicher kein Magic-Angle-Phäno- } \\
\text { men an der Supraspinatussehne zu haben }\end{array}$ \\
\hline \multicolumn{3}{|l|}{ cor T1 (evtl mit fs) } \\
\hline sag T1, evtl. PD/T2 & $\begin{array}{l}\text { senkrecht zu cor, parallel zum Humerusschaft; bis zum Sca- } \\
\text { pula-Y nach medial messen }\end{array}$ & $\begin{array}{l}\text { nicht fettsättigen, um fibröses Gewebe im Rotatoren- } \\
\text { intervall nicht zu übersehen }\end{array}$ \\
\hline ggf. axial GRE & & zur Beurteilung des Labrums \\
\hline ABER Position: T1 fs & $\begin{array}{l}\text { koronares Topo, darauf senkrecht parallel zum Humerus- } \\
\text { schaft planen, der Schnitt geht schräg von hinten oben nach } \\
\text { vorne unten durch das Glenoid }\end{array}$ & $\begin{array}{l}\text { bei Frage nach dem anterior-inferioren Labrum und } \\
\text { dem posterior-superioren Impingement }\end{array}$ \\
\hline
\end{tabular}


Ellbogen

Lagerung

\begin{tabular}{|l|l|l|}
\hline Sequenz & Planung & Kommentar \\
\hline ax PD fs & senkrecht zum Humerus und Ulna planen & $\begin{array}{l}\text { Ellbogen möglichst gestreckt messen, z. B. in der } \\
\text { Kniespule; } \\
\text { weit genug nach distal bis zum Ansatz der Bizeps- } \\
\text { sehne messen } \\
\text { gebeugten Ellbogen in Schulterspule messen }\end{array}$ \\
\hline ggf. ax PD/T2 & & \\
\hline cor T1 & parallel zum Humerus und Ulna und auf der axialen & \\
\hline cor PD fs & parallel zur Vorderkante Humerus planen & \\
\hline sag PD/ T2 & senkrecht auf cor planen & $\begin{array}{l}\text { weit genug nach distal bis zum Ansatz der Bizeps- } \\
\text { sehne messen }\end{array}$ \\
\hline ggf. i. v. KM & & weit genug nach distal bis zum Ansatz der Bizeps- \\
\hline ax T1 fs & & sehne messen \\
\hline cor oder sag T1 fs & & Fov bis zum Ansatz der Bizepssehne planen \\
\hline
\end{tabular}

Handgelenk (KM)

Lagerung Bauchlage, Kopf voran, Arm in Supermannposition.

\begin{tabular}{|l|l|l|}
\hline Sequenz & Planung & Kommentar \\
\hline cor T1 & & wichtig für Knochenmarksignal \\
\hline cor PD fs & & $\begin{array}{l}\text { wichtig für Knochenmarködem bei Knochennekrose } \\
\text { oder okkulten Fraktur }\end{array}$ \\
\hline ax T2 & schön für Ganglien \\
\hline sag PD (alternativ T1) & & bei Frage Nekrose oder Fraktur eher T1 \\
\hline \begin{tabular}{l|l} 
evtl. cor GRE \\
(z. B. TRUFI WEX)
\end{tabular} & sehr schöne Knorpelsequenz \\
\hline i. v. KM & & erhöht die Sensitivität für Bandverletzungen und ist \\
\hline cor T1 fs & & \\
\hline ax T1 fs & & \\
\hline
\end{tabular}

Handgelenk nativ

(bei Frage nach okkulten Frakturen oder einer frischen Bandverletzung).

\begin{tabular}{|l|l|l}
\hline Sequenz & Planung & Kommentar \\
\hline cor T1 & & wichtig für Knochenmarksignal und Frakturlinie \\
\hline cor PD fs & & $\begin{array}{l}\text { wichtig für Knochenmarködem bei Knochennekrose oder } \\
\text { okkulten Fraktur und frischen Bandverletzungen }\end{array}$ \\
\hline ax PD fs & $\begin{array}{l}\text { wichtig für Knochenmarködem bei Knochennekrose oder } \\
\text { okkulten Fraktur und frischen Bandverletzungen }\end{array}$ \\
\hline sag PD fs & $\begin{array}{l}\text { wichtig für Knochenmarködem bei Knochennekrose oder } \\
\text { okkulten Fraktur und frischen Bandverletzungen }\end{array}$ \\
\hline evtl. cor TRUFI WEX & sehr schöne Knorpelsequenz \\
\hline
\end{tabular}


Handgelenk Arthro

\begin{tabular}{|l|l|l|}
\hline Sequenz & Planung & Kommentar \\
\hline cor T1 fs & & wichtig für Bandverletzungen mit KM Übertritt \\
\hline cor T2 fs & wichtig für Knochenmarködem und Bandverletzungen \\
\hline ax T1 & wichtig für Bandverletzungen mit KM Übertritt & \\
\hline ax PD fs & & sehr schöne Knorpelsequenz \\
\hline sag PD fs & & \\
\hline $\begin{array}{l}\text { evtl. cor 3 D GRE } \\
\text { (z. B. 3 D VIBE fs) }\end{array}$
\end{tabular}

\section{Untere Extremität}

Hüfte

\begin{tabular}{|l|l|l|}
\hline Sequenz & Planung & Kommentar \\
\hline $\begin{array}{l}\text { Übersicht über beide Hüften } \\
\text { cor oder ax T2/PD fs, } \\
\text { alternativ T2 STIR }\end{array}$ & $\begin{array}{l}\text { streng axial ca. } 2 \mathrm{~cm} \text { oberhalb des Hüftgelenks } \\
\text { bis ca. } 2 \mathrm{~cm} \text { unterhalb der Sitzbeine } \\
\text { streng koronar von Symphyse bis ISG-Fugen }\end{array}$ & $\begin{array}{l}\text { Füße etwas nach innen drehen, ggf. zusam- } \\
\text { mentapen; kann mit } 5 \text { mm Schichtdicke ge- } \\
\text { messen werden; dient für Schmerzursache au- } \\
\text { Berhalb des Hüftgelenks }\end{array}$ \\
\hline ab jetzt einseitig messen & & \\
\hline cor T1 & streng cor & \\
\hline cor PD fs & parallel zum Schenkelhals & gut zur Beurteilung des Knorpels \\
\hline schräg ax PD (evtl mit fs) & & die TRUFI Water Excitation ist eine sehr schöne \\
\hline sag PD & & Knorpelsequenz \\
\hline ggf. sag trufi WEX & & \\
\hline ggf. cor und tra T1 fs KM & & \\
\hline
\end{tabular}

Hüfte Arthro

\begin{tabular}{|c|c|c|}
\hline Sequenz & Planung & Kommentar \\
\hline $\begin{array}{l}\text { Übersicht über beide Hüften } \\
\text { cor oder ax T2/PD fs, } \\
\text { alternativ T2 STIR }\end{array}$ & $\begin{array}{l}\text { streng axial ca. } 2 \mathrm{~cm} \text { oberhalb des Hüftgelenks bis } \\
\text { ca. } 2 \mathrm{~cm} \text { unterhalb der Sitzbeine } \\
\text { streng koronar von Symphyse bis ISG-Fugen }\end{array}$ & $\begin{array}{l}\text { Füße etwas nach innen drehen, ggf. zusammentapen; kann mit } \\
5 \text { mm Schichtdicke gemessen werden; dient für Schmerzursache } \\
\text { außerhalb des Hüftgelenks }\end{array}$ \\
\hline \multicolumn{3}{|l|}{ einseitig messen } \\
\hline cor T1 (evtl mit fs) & streng cor & \\
\hline \multicolumn{3}{|l|}{ cor PD fs } \\
\hline schräg ax T1 fs & parallel zum Schenkelhals & \\
\hline \multicolumn{3}{|l|}{ sag T1 fs } \\
\hline ggf. sag TRUFI WEX & & die TRUFI Water Excitation ist eine sehr schöne Knorpelsequenz \\
\hline \multicolumn{3}{|l|}{ radial } \\
\hline ggf. cor T1 GRE 3 D WEX & \multicolumn{2}{|c|}{$\begin{array}{l}\text { werden radiale Schichten gemessen sollte zuerst eine koronare 3D-Sequenz gemessen werden, um darauf die radialen } \\
\text { Schichten zu planen }\end{array}$} \\
\hline PD radial (alternativ $\mathrm{T} 1 \mathrm{fs}$ ) & & \\
\hline
\end{tabular}


Knie

\begin{tabular}{|c|c|c|}
\hline \multicolumn{2}{|l|}{ Sequenz } & Planung \\
\hline cor T1 & parallel zu den Hinterkanten der Femurkondylen & $\begin{array}{l}\text { Kommentar } \\
\text { tal planen, das Innenband setz } \\
\text { tibae an }\end{array}$ \\
\hline cor PD fs & senkrecht zu cor, parallel zur Beinachse & \\
\hline \multicolumn{3}{|l|}{ sag PD fs } \\
\hline (sag PD o. T2) & & $\begin{array}{l}\text { gut zur Beurteilung des Hoffa Fettkörpers und des suprapa- } \\
\text { tellaren Recessus }\end{array}$ \\
\hline ax PD fs & $\begin{array}{l}\text { auf der cor und sag exakt parallel zum Tibiaplateau; ca. } 2 \mathrm{~cm} \\
\text { proximal der Patella bis Fibulaköpfchen }\end{array}$ & \\
\hline ggf. KB Sequenz T2 & schräg ax parallel zum VKB und/oder senkrecht zum VKB & TE > 70 ms und keine Fettsättigung \\
\hline ax und cor o. sag T2 GRE & & bei V.a. PVNS \\
\hline ax und cor o. sag $\mathrm{T} 1 \mathrm{fs} \mathrm{KM}$ & & bei Frage nach Entzündungen \\
\hline
\end{tabular}

Sprunggelenk

\begin{tabular}{|l|l|l|}
\hline Sequenz & Planung & Kommentar \\
\hline ax PD(fs) & $\begin{array}{l}\text { in beiden Ebenen senkrecht auf die Tibia; oberhalb der } \\
\text { Syndesmose beginnen bis Ende Calcaneus }\end{array}$ & $\begin{array}{l}\text { mit axial beginnen und cor und sag auf den axialen Schichten } \\
\text { planen }\end{array}$ \\
\hline cor T1 & exakt cor zum OSG planen, parallel zur Tibia & unten den Calcaneus einschließlich Plantarfaszie im FOV \\
\hline cor PD (fs) & & unten den Calcaneus einschließlich Plantarfaszie im FOV \\
\hline sag PD fs & senkrecht zu cor, parallel zum Tibiaschaft & Innenband \\
\hline & & höchstens 9 Schichten \\
\hline ggf. schräg ax PD (fs) & axial schräg im Verlauf der Syndesmose planen & gut für die Beurteilung der Peroneal- und Fußbeugersehnen \\
\hline ggf. schräg cor PD fs & Verlauf ist etwa Innenkurve Rist -> Spitze Calcaneus & bei chronischen Schmerzen, V. a. entzündlichen Veränderungen \\
\hline ggf. i. v. KM & & \\
\hline ax T1 fs & & \\
\hline cor oder sag T1 fs & & \\
\hline & & \\
\hline
\end{tabular}

\section{Autor}

Prof. Dr. med. Rolf Janka

Universität Erlangen

\section{Korrespondenzadresse}

Prof. Dr. med. Rolf Janka

Heinrich-Franke-Weg 15

91052 Erlangen 